\title{
Comment on some proposed mechanisms for attenuation of third sound*
}

\author{
M. W. Cole \\ University of Washington, Seattle, Washington 98195 \\ D. L. Goodstein \\ California Institute of Technology, Pasadena, California 91109
}

(Received 25 May 1973)

\begin{abstract}
Two explanations have been proposed for an apparent discrepancy between theoretical prediction and experimental measurement of third-sound attenuation. One of these proposes a new "macroscopic quantum uncertainty principle," and the other proceeds via nonlinear, anharmonic effects due to zero-point fluctuations. We argue that neither suggestion is acceptable.
\end{abstract}

Recently considerable attention has been focused on the attenuation of third sound in superfluid $\mathrm{He}$ films. Predictions of this attenuation by Bergman ${ }^{1}$ which are based on the linearized hydrodynamic equations of the film-gas system disagree with experimental data of Wang and Rudnick. ${ }^{2}$ This has prompted two sorts of explanation ${ }^{3,4}$ which we discuss below. We regard neither of these as acceptable.

Putterman et al., ${ }^{3}$ have conjectured that the "missing" attenuation arises from a proposed "macroscopic quantum uncertainty principle," analogous to the microscopic Heisenberg principle. The argument is that there exists a quantum uncertainty in the component of superfluid velocity perpendicular to the plane of the substrate given by

$$
\delta v_{s} \approx \hbar / m Z \text {, }
$$

where $m$ is the mass of a helium atom, and $Z$ the film thickness. There is extra attenuation whenever the speed of third sound, $c_{3}$, falls below this velocity, i.e., when

$$
c_{3}<\hbar / m Z
$$

(the authors insert a factor $\sqrt{12}$ which is unimportant for the present argument). The uncertainty in the perpendicular component of $v_{s}$ affects third sound, which propagates parallel to the plane of the film, because it introduces an uncertainty $\sim(\hbar / m Z)^{2}$ into the quantity $\left(\mu+\frac{1}{2} v_{s}^{2}\right)$, where $\mu$ is the chemical potential per unit mass. The quantity $\left(\mu+\frac{1}{2} v_{s}^{2}\right)$ is the driving potential of the film.

The meaning of the conjecture is that in any experiment in which $Z$ is known, the potential in the film cannot be measured more accurately than

$$
(\hbar / m Z)^{2} \text {. }
$$

For saturated films, where Eq. (2) is satisfied, the uncertainty in the potential is at least

$$
\delta\left(\mu+\frac{1}{2} v_{s}^{2}\right) \approx c_{3}^{2} .
$$

But for a saturated film, $c_{3}^{2} \approx 3\left(\rho_{s} / \rho\right) g y$ where $\rho_{s} / \rho$, the superfluid fraction, is $\sim 1$ at low temperature, and $g y$ is the gravitational potential a distance $y$ above the bath. Thus the conjecture predicts the film potential to have a quantum uncertainty of the order of the gravitational potential itself,

$$
\delta\left(\mu+\frac{1}{2} v_{s}^{2}\right) \approx g y .
$$

The evidence to the contrary is among the best established of experimental facts concerning superfluid helium. The very term superfluid refers to the ability of the liquid to flow at accurately uniform potential. The actual experimental uncertainty in the potential is orders of magnitude smaller than that required by Eq. (4). ${ }^{5}$

We note also in passing that previous treatments ${ }^{6}$ of quantum fluctuations in $v_{s}$ have found those to be unimportant relative to thermal fluctuations except at very low temperatures. If one does wish to consider the possibility of fluctuations of the type proposed in Eq. (1), the result, $\hbar / m Z$, should be compared to the relevant thermal value $\left(k_{B} T / m\right)^{1 / 2}$, where $k_{B}$ is Boltzmann's constant. The latter dominates except at very low $T$ or in the thinnest unsaturated films. Finally, a more conventional ${ }^{7}$ estimate of the uncertainties in the perpendicular component of $v_{s}$ is obtained by relating it to the uncertainty in the phase $\varphi$ of the condensate wave function, across the film. Since $v_{s}=(\hbar / m) \nabla \varphi$,

$$
\begin{aligned}
& \delta v_{s} \approx \frac{\hbar}{m} \frac{\delta \varphi}{Z} \approx \frac{\hbar}{m Z} \frac{1}{\delta N}, \\
& \delta \varphi \delta N \sim 1,
\end{aligned}
$$

where $\delta N$ is the quantum uncertainty in the particle number. A reasonable estimate for a third-sound experiment would be $\delta N \approx \sqrt{N}$ where $N \approx \rho \lambda^{2} Z / m, \rho$ 
being the mass density and $\lambda$ the third-sound wavelength. This yields a $\delta v_{s}$ many orders of magnitude smaller than Eq. (1). The appearance of $\delta N$ in the denominator of Eq. (5) emphasizes the collective nature of the quantity $v_{s}$, a property that has evidently been neglected in formulating Eq. (1).

We thus conclude that the conjecture is at variance with the main body of both experimental and theoretical understanding of superfluidity.

An alternative explanation of the discrepancy between third-sound theory and experiment has been proposed by Chester and Maynard. ${ }^{4}$ The latter have attempted to show that anharmonic terms entering the third-sound effective Hamiltonian cause nonlinearities and hence attenuation in third-sound propagation. As pointed out by the authors, the effect proceeds by way of a large mean square displacement of the film-vapor interface produced by zero-point fluctuations in the third-sound modes. They conclude that the rms fluctuation, or surface width, is or order of onethird the film thickness.

The large surface fluctuation and consequent nonlinearities are produced entirely by veryshort-wavelength modes, where the approximations used are quite incorrect. Physically, small amplitudes in these modes are assured by surface- tension forces, which have been neglected. In particular, a term,

$$
-\nabla\left(\alpha \nabla^{2} n / \rho^{2}\right) \text {, }
$$

where $\alpha$ is the surface tension and $n$ the mass per unit area, should be added to the left-hand side of Eq. (5) of Ref. 4. The dispersion relation that results is

$$
\begin{aligned}
& \omega=c_{3} q\left(1+a^{2} q^{2}\right)^{1 / 2}, \\
& a^{2} \cong \alpha / \rho f .
\end{aligned}
$$

Here $f$ is the van der Waal's force per unit mass at the film surface, $c_{3}$ the speed of third sound, and $q$ the wave number. The correction term in parenthesis in Eq. (8) becomes important for $q \approx a^{-1} \approx 10^{-3}-10^{-2} \AA^{-1}$, whereas Chester and Maynard obtain their effect by applying the uncorrected formula up to $q \approx 1 \AA^{-1}$. When the surface tension is properly included, the interfacial diffusiveness is restored to a value of the order of the interatomic spacing, and the anharmonic contribution to third-sound attenuation becomes negligible. ${ }^{8}$

Finally, we remark that the present hydrodynamic theory of third sound is sufficiently approximate that it is premature to conclude that such an approach cannot succeed.
* This work was supported in part by grants from the National Science Foundation, and one from the Research Corporation (DLG).

${ }^{1}$ D. J. Bergman, Phys. Rev. A $\underline{3}, 2058$ (1971).

${ }^{2}$ T. G. Wang and I. Rudnick, J. Low Temp. Phys. $\underline{9}, 425$ (1972).

${ }^{3}$ S. J. Putterman, R. Finkelstein, and I. Rudnick, Phys. Rev. Lett. 27, 1697 (1971); S. J. Putterman, in Proceedings of the Thirteenth International Conference on Low Temperature Physics (Univ. of Colorado Press, Boulder, Colo., 1973).

${ }^{4} \mathrm{M}$. Chester and R. Maynard, Phys. Rev. Lett. $\underline{29}$, 628 (1972).

${ }^{5}$ This is particularly clear in an experiment of W. E. Keller, where $Z$ and the potential were measured simultaneously. See W. E. Keller, Phys. Rev. Lett. $\underline{24}, 569(1970)$.

${ }^{6}$ R. A. Ferrell, N. Menyhàrd, H. Schmidt, F. Schwabl, and P. Szépfalusy, Ann. Phys. (N.Y.) 47, 565 (1968); P. C. Martin in Statistical Mechanics at the Turn of the Decade, edited by E. G. D. Cohen (Dekker, New York, 1971).

${ }^{7}$ P. W. Anderson, Rev. Mod. Phys. 28, 298 (1966).

${ }^{8}$ For the surface width of the bulk liquid, see M. W. Cole, Phys. Rev. A 1 , 1838 (1970). The dispersion relation, Eq. (8), should reduce to the ordinary capillary wave formula when $q \gg Z^{-1}$, but that is far outside the domain of applicability of the present approach. 